\title{
Discovery of multi-seeded multi-mode formation of embedded clusters in the Rosette Molecular Complex
}

\author{
Jin Zeng Li ${ }^{1,2} \&$ Michael D. Smith ${ }^{2}$ \\ ${ }^{1}$ National Astronomical Observatories, Chinese Academy of Sciences, Beijing 100012, \\ China (E-mail: ljz@bao.ac.cn) \\ ${ }^{2}$ Armagh Observatory, College Hill, Armagh BT61 9DG, N. Ireland, UK
}

\begin{abstract}
An investigation based on data from the spatially complete 2MASS Survey reveals that a remarkable burst of clustered star formation is taking place throughout the south-east quadrant of the Rosette Molecular Cloud. Compact clusters are forming in a multi-seeded mode, in parallel and at various places. In addition, sparse aggregates of embedded young stars are extensively distributed. In this study, we report the primary results and implications for high-mass and clustered star formation in giant molecular clouds. In particular, we incorporate for the first time the birth of medium to low-mass stars into the scenario of sequential formation of $\mathrm{OB}$ clusters. Following the emergence of the young $\mathrm{OB}$ cluster NGC 2244, a variety of manifestations of forming clusters of medium to high mass appear in the vicinity of the swept-up layer of the HII region as well as further into the molecular cloud. The embedded clusters appear to form in a structured manner, which suggests they follow tracks laid out by the decay of macroturbulence. We address the possible origins of the turbulence. This leads us to propose a tree model to interpret the neat spatial distribution of clusters within a large section of the Rosette complex. Prominent new generation OB clusters are identified at the root of the tree pattern.
\end{abstract}

Subject headings: Infrared: stars - ISM: clouds - Stars: formation - Stars: premain sequence

\section{Introduction}

Giant Molecular Clouds (GMCs) are the primary sites of star birth in the Galaxy. In particular, they appear essential for the formation of massive stars in OB associations. The Rosette Molecular Cloud (RMC), residing in the constellation of Monoceros, is situated in 
the Perseus arm of the Galaxy. It has an extent of around 100 pc (Dorland \& Montmerle 1987 ) and contains $\sim 10^{5} \mathrm{M} \odot$ of gas \& dust (Blitz \& Thaddeus 1980). It is among the most massive GMCs in the Galaxy and is associated with one of the most outstanding regions of massive star formation.

The Rosette Nebula is located at the north-west tip of the GMC. It is a spectacular HII region of $\sim 30 \mathrm{pc}$ in scale, famous for harboring one of the youngest $\left(\sim 3 \times 10^{6}\right.$ yrs, Ogura \& Ishida 1981) open clusters, NGC 2244, and is itself being excavated by the strong stellar winds from the immersed OB stars at the center. The favorable orientation of the Rosette Nebula, perpendicular to the line of sight, and its prominent interaction with the ambient molecular cloud, provides an excellent opportunity to test the theory of sequential formation of OB clusters in GMCs (Elmegreen \& Lada 1977; Lada 1987).

NGC 2244 is, on the other hand, located roughly at the center of the Mon OB2 association, which has a physical scale of up to 280 pc (Blaauw 1964; Singh \& Naranan 1979). It represents the youngest subgroup of the $\mathrm{OB}$ association and extends $\sim 20$ pc. Another two subgroups of OB stars were identified and ages of the order of $\sim 5 \times 10^{6}$ yrs and a few $10^{7}$ yrs suggested (Turner 1976). One of their members could have exploded into a type II supernova and formed a supernova remnant (SNR), the Monoceros Loop (Davies, 1963; Aharonian et al. 2004). However, the SNR has a dynamical age of only $3-15 \times 10^{4} \mathrm{yrs}$, and the shock front has probably just reached the edge of the Rosette Nebula and is, therefore, unlikely to be responsible for any on-going star formation activity in the RMC (Blitz \& Thaddeus 1980; Townsley et al. 2003). Nevertheless, the overall picture is that GMCs in the galactic disk are transitory objects, subject to sequential cluster formation.

The RMC is highly clumped, as has been established through various observations from mid-infrared to radio wavelengths (Cox, Deharveng \& Lenne 1990; Blitz \& Stark 1986; Williams, Blitz \& Stark 1995; Shipman \& Carey 1996; Kuchar \& Bania 1993; Celnik 1985). It could be producing new generations of young stars and embedded clusters in its densest rim (Cox et al. 1990; Block, Geballe \& Dyson 1993; Carpenter, Snell \& Schloerb et al. 1993; Hanson, Geballe \& Conti et al. 1993; Phelps 1994). Patches of intensive X-ray emission spatially associated with the dense clumps, which is indicative of active star formation, were detected by Gregorio-Hetem, Montmerle and Casanova et al. (1998).

Phelps \& Lada (1997) have spotted, via visual inspection of near-infrared images, 7 aggregates of infrared stars, each associated with an IRAS point source. This demonstrated that star formation is continuing in this area in a distributed manner and different star formation mechanisms may be at work. Unfortunately, a complete, unbiased census of star formation over the entire spatial extent of the RMC is not yet available. However, the 2MASS JHKs data permit us to identify and analyze considerably more embedded objects. 
A detailed, in-depth study will help to elucidate to what extent star or cluster formation is proceeding and provide clues as to what mechanisms are governing the star birth processes across this exceptional area. This Letter explores a panorama of clustered star formation in the RMC. Detailed studies of each well-defined region of cluster formation will be presented in a forthcoming series. In addition, the first infrared study of the young massive cluster, NGC 2244, resolved into a combination of a 'twin' cluster and a relic arc of slightly reddened young stars, will be discussed elsewhere (Li 2005).

\section{Data Acquisition and Analysis}

Archived data from the 2MASS Point Source Catalog (PSC) and IRAS Sky Survey Atlases (ISSA) were retrieved via IRSA (http://irsa.ipac.caltech.edu/). IRAS HIRES images of the RMC were subsequently obtained by the email requesting system running at the IPAC data center. The following sample selection criteria were employed to guarantee the reliability of the 2MASS data in use. (1) Each source extracted from the 2MASS PSC must have a certain detection in all three of the J, H, and Ks bands. (2) We restrict the Ks band signal to noise ratio to values above 15 to tightly constraint field stars in the control field to the main sequence loci on the (J-H) to (H-Ks) diagram (Li \& Smith 2005a). However, the flux and resolution limited 2MASS survey, and our stringent selection criteria, will in combination result in drawbacks. Firstly, the number of proto-OB stars that are deeply embedded can be underestimated. This is likely to occur in the densest regions with the highest extinction. Secondly, this work is limited to the study of comparatively high mass cloud members. With a $90 \%$ completeness limit to $\sim 0.8 \mathrm{M} \odot$ objects, many disk stars with masses above this limit can be trimmed off.

\section{Results and Discussion}

Even in a single near-infrared band, two separate regions of significant stellar density enhancement are readily identified. One of these stellar concentrations coincides with the young open cluster NGC 2244 and the other corresponds spatially to the densest rim of the RMC. The latter has no known optical counterparts and is therefore likely to be a recently formed embedded, compact cluster.

However, when this distribution is plotted as a function of H-Ks color, fine structure signifying large scale clustering becomes evident throughout the south-east quadrant (Fig. 1). Positions of previously identified embedded clusters and young massive stars are overplotted 
in Fig. 1. These are spatially associated with the majority of the stellar concentrations in the plot, indicating the young, embedded nature of the clustering of reddened sources. The candidate embedded clusters are further divided into four major groups A, B, C \& D, partially according to their distinct concentrated appearance and their association with dense clumps as outlined by the IRAS $100 \mu \mathrm{m}$ emission (Cox et al. 1990) and CO detections (Blitz \& Thaddeus 1980; Williams et al. 1995). The majority of the candidate young stars are either congregated within the outlined regions in Fig. 1 or appear as minor stellar aggregates not identified in this paper. Only a small fraction of the sources identified here with heavy line of sight extinction are found to be in a wide scattered distribution and are to be attributed to isolated formation.

Color-color diagrams of each of the four regions are presented in Fig. 2. About one sixth to a fourth of the 2MASS sources in each region are found to be located to the right of the reddening band of normal field stars. These sources thus possess intrinsic color excesses originating from emission from circumstellar dust, commensurate with their embedded nature. A concentration of background giants along the left edge of the reddening band directly above the giants track is apparent on the color-color diagram for at least Region D, which covers the largest area in the RMC. This indicates an inhomogeneous distribution of extinction for the cloud that ranges mainly between 2-10 mag. In the densest clumps, however, visual extinction can even exceed $25 \mathrm{mag}$. This confirms the very clumpy nature of the molecular cloud and is consistent with a high clump to interclump density contrast (Schneider et al. 1998).

We attempt to eliminate foreground sources, which possess a typical color of $(\mathrm{H}-\mathrm{Ks}) \sim$ $0.2 \mathrm{mag}$ based on a detailed investigation of the reference fields (Li \& Smith 2005a). Apparent background giants concentrated within an elliptical area on the color-color diagrams can also be excluded. The fraction of excess emission sources then remaining in each region amounts to between one third and a half. This provides convincing evidence that the majority of the reddened sources are embedded young stars still associated with their parental clouds. We thus confirm the unprecedented widespread presence of forming clusters embedded in the RMC.

A close correspondence of the compact subclusters in each region with the distribution of the most luminous IRAS sources (Cox et al. 1990) and the densest clumps (Williams et al. 1995) is clearly shown in Fig. 1. This suggests a 'multi-seeded' origin of the embedded clusters in the fragmented interaction layer of the HII region and at various places deep into the molecular complex. The term multi-seeded is introduced to describe a situation in which the formation of clusters in distinct clumps in a distributed manner is proceeding in parallel. On the other hand, clustered star formation is taking place both in isolation in the swept-up 
shells and in a more structured mode further away from NGC 2244. The structured mode surprisingly continues down to the south-east boundary of the RMC.

The embedded nature of a source is thought to be fully substantiated if it meets the following two criteria. These are (1) an intrinsic color excess in the near infrared i.e. $\mathrm{E}(\mathrm{H}-$ $\mathrm{Ks})>0$ and $(2)$ a $(\mathrm{H}-\mathrm{Ks})$ color of greater than 0.2 . This again helps to exclude possible contamination from the foreground. In this manner, some 30 excessive emission sources were removed from our sample. A clear density enhancement of embedded young stars toward the outlined regions remains as evidence for a non-random distribution of young stellar objects across the extincted areas. In addition, the surface density of excess emission sources ranges from an average of $0.55 \mathrm{pc}^{-2}$ over the entire extent of the RMC to $\sim 23 \mathrm{pc}^{-2}$ for the compact subclusters in Region $\mathrm{C}$ where the process of star formation is probably still going on.

An investigation of the properties of each embedded cluster was carried out and the results are summarized in Table 1 . The analysis included the construction of near infrared color-color and color-magnitude diagrams and the calculation of the Ks Luminosity Function (KLF). An example color-magnitude diagram for objects in the Region $\mathrm{C}$ cluster is presented in Fig. 3. Both components of the known binary system AFGL 961 are identified as candidate proto-O stars (for a detailed analysis, see Li \& Smith, 2005b) and are indicated with asterisks. The east component of AFGL 961, presumably a single object, is shown to have a possible mass of up to $\sim 130 \mathrm{M} \odot$. It is probably one of the most massive protostars forming in the complex. A nearby progenitor O star, represented on the diagram with a solid square, may prove to be a third component of the system because of its close proximity to AFGL 961 within a projected distance of 0.2 pc ( $\mathrm{Li} \&$ Smith 2005b).

The clusters embedded in Regions A \& B are spatially coincident with the fragmented arcs of the swept-up layer (Fig. 1 \& 4a) of the HII region associated with NGC 2244, an emerging young open cluster known to have a turn-off age of $\sim 2$ Myrs (Park \& Sung 2002). Based on a KLF study of the Regions A \& B clusters, an age estimate of $\sim 1$ Myr is obtained by comparing to KLFs of embedded clusters with known ages (Lada \& Lada 1995). At a distance of $\sim 20$ pc away from the center of NGC 2244, the age of the shell clusters in Regions $\mathrm{A} \& \mathrm{~B}$ is consistent with probably their triggered nature by the shocked wave associated with the ionization front of the HII region, which propagates at a velocity of $\sim 20 \mathrm{~km} \mathrm{~s}^{-1}$ (Smith 1973).

No prominent age spread between the sub-clusters or aggregates in Regions C \& D can be quantitatively addressed based on a study of the KLF. However, we suggest that the group of proto-O stars associated with AFGL 961, situated in the south-east of Region C (Fig. 1), is probably among the latest episodes of star and cluster formation. Due to their estimated high masses and their protostellar origin, this proto-O star system may have ages 
of less than a few $10^{4}$ yrs (Hanson et al. 1993). At a distance of $\sim 15$ pc from the interface with the Rosette Nebula, its location in the densest ridge of the RMC probably signifies the gestation of new generation OB clusters following the emergence of NGC 2244.

\section{Structured clustering of star formation in the RMC}

As a comparison, we have derived the distributions of optical depth at $100 \mu \mathrm{m}$ and color temperature based on the IRAS ISSA (Fig. 4a \& b, for an introduction of the methods used, see Li \& Chen 1996). The outlined regions A \& B are spatially coincident with the sweptup layer of the Rosette Nebula, and Region $\mathrm{C}$ with the densest rim of the RMC. However, Region $\mathrm{D}$ is found through the plots to have comparatively lower extinction (Fig. 4a), in agreement with results from the $\mathrm{CO} \& \mathrm{C}^{13} \mathrm{O}$ detections (Blitz \& Stark 1986; Williams et al. 1995). The dust temperatures, however, are between 25 and $41 \mathrm{~K}$ (Fig. 4b), comparable to other regions of cluster formation.

Star formation is evidenced by our study to be occurring in an extensive and regulated way across the south-east section of the RMC. This refines the picture introduced by Cox et al. (1990). It is taking place within a selected spread but not all of the clumpy area of Region D (Fig. 4a), as is illustrated by the stellar distribution and the overplotted continuous lines in Fig. 5. Nevertheless, it follows a structured pattern that first developed along the major axis of the RMC, in parallel to the Perseus Arm in the Galactic plane (Fig. 5). The pattern, resembling a 'tree' in structure, is related to the gas filaments of the cloud (Williams et al. 1995), but does not seem to break where the clumps or filaments do. This indicates the existence of some underlining tracks.

A schematic model is thus invoked to interpret the widespread star and cluster formation activity in Regions C \& D of the RMC and, perhaps, the south-east half of the complex as a whole. Large scale turbulence is suggested to originate from the spiral density wave of the Galaxy (Woodward 1976) or tidal force dissipation of the clouds in the Perseus arm. Additional turbulence is injected from internal sources, through a combination of feedback

effects from the young massive stars in NGC 2244 and Region C, especially the violent outflow activity related to the formation of the proto-O stars associated with AFGL 961 (Lada \& Gautier 1982). The perturbations trigger the collapse of clumps, and star formation consequently occurs along tracks following the decay of the macroturbulence, tracing out a tree pattern.

In the frame of the proposed tree model, the rich massive cluster embedded in Region $\mathrm{C}$ is spatially coincident with the bulk of the CO emission of the complex (Blitz \& Thaddeus 
1980; Blitz \& Stark 1986; Williams et al. 1995) and constitutes the root of the tree. It harbors the most massive protostars in the complex and perhaps also among those known in the Galaxy. Medium mass clusters and loose aggregates with stellar masses of up to $\sim 50$ $\mathrm{M} \odot$ are developing down to the south-east boundary of the complex. These correspond to the branches. This scenario is supported by simulations of decaying supersonic turbulence, in which the decay follows a period of energy pumping and compression (Mac Low \& Klessen 2004), but in this case it corresponds to wider or even galactic scales.

This extensive study of the RMC has, for the first time, incorporated into the scenario of sequential formation of OB clusters in GMCs the involvement of medium to low mass stars which, in combination, reveal a more complex nature. We argue that the history of cluster formation associated with the RMC, though in a sequential manner both in space and time, is better interpreted in terms of sequential collapse of dense clumps along the major axis of the GMC. The collapsed regions outline tracks of the decay of macroturbulence rather than having been exclusively triggered by the interference of former generations of OB clusters of the Mon OB2 association.

\section{Acknowledgments}

We are grateful to an anonymous referee for constructive comments which improved the scientific presentation of the paper. We thank Prof. L. Blitz for going through the manuscript and providing very useful comments. J. Z. Li acknowledges help from Dr. J. P. Williams for making available his original $\mathrm{CO} \& \mathrm{C}^{13} \mathrm{O}$ data of the RMC. This publication makes use of data products from the Two Micron All Sky Survey, which is a joint project of the University of Massachusetts and the Infrared Processing and Analysis Center/California

Institute of Technology, funded by the National Aeronautics and Space Administration and the National Science Foundation. This work also makes use of the IRAS PSC and ISSA data. This project is supported by SRF for ROCS, SEM. Finally, we acknowledge funding from the Particle Physics and Astronomy Research Council, UK, and the Department of Culture, Arts and Leisure, Northern Ireland.

\section{REFERENCES}

Aharonian, F. A. et al. 2004, A\&A 417, 973

Bessel, M. S. \& Brett, J. M., 1988, PASP 100, 1134

Blaauw, A., 1964, ARA\&A 2, 213

Blitz, L. \& Stark, A. A., 1986, ApJ 300, L89 
Blitz, L. \& Thaddeus, P., 1980, ApJ 241, 676

Block, D. L., Geballe, T. R. \& Dyson, J. E., 1993, A\&A 273, L41

Carpenter, J. M., Snell, R. L., Schloerb, F. P. \& Strutskie, M. F. 1993, ApJ 407, 657

Celnik, W. E., 1985, A\&A 144, 171

Cox, P., Deharveng, L. \& Leene, A., 1990, A\&A 230, 181

Davies, R. D., 1963, Observatory 83, 172

Dorland, H. \& Montmerle, T., 1987, A\&A 177, 243

Elmegreen, B. G., \& Lada, C. J., 1977, ApJ 214, 725

Hanson, M. M., Geballe, T. R., Conti, P. S. \& BLock, D. L., 1993, A\&A 273, L44

Kuchar, T. A. \& Bania, T. M., 1993, ApJ 414, 664

Lada, C. J., 1987, IAUS 115, 1

Lada, C. J. \& Gautier III, T. N., 1982, ApJ 261, 161

Lejeune, T. \& Schaerer, D., 2001, A\&A 366, 538

Li, J. Z., 2005, ApJ 625, in press

Li, J. Z. \& Smith, M. D., 2005a, A\&A 431, 925

Li, J. Z. \& Smith, M. D., 2005b, AJ 129, in press

Li, J. Z. \& Chen, P. S., 1996, Chinese Astro. \& Astroph. 20, 445

Mac Low, M. \& Klessen, R. S. 2004, Reviews of Modern Physics, 76, 125

Meyer, M. R., Calvet, N., Hillenbrand, L. A., 1997, A\&A, 114, 288

Ogura, K. \& Ishida, K., 1981, PASJ 33, 149

Park, B.-G., \& Sung, H. 2002, AJ 123, 892

Phelps, R. L., 1994, in The Nature and Evolutionary Status of Herbig Ae/Be stars, ed. P. S. The, M. R. Perez \& P. J. van den Heuvel (San Francisco:ASP), 339

Phelps, R. L. \& Lada, E. A., 1997, ApJ 477, 176 
Rieke, G. H. \& Lebofsky, M. J., 1985, ApJ 288, 618

Schneider, N., Stutzki, J., Winnewisser, G. et al., 1998, A\&A 338, 262

Shipman, R. F. \& Carey, S. T., 1996, ApJ 469, L131

Singh, K. P. \& Naranan, S., 1979, Ap\&SS 66, 191

Smith, M. G., 1973, ApJ 183, 111

Townsley, L. K., et al. 2003, ApJ 593, 874

Turner, D. G., 1976, ApJ 210, 65

Williams, J. P., Blitz, L., Stark, A. A., 1995, ApJ 451, 252

Woodward, P. R., 1976, ApJ 207, 484 
Fig. 1. - The spatial distribution of the 2MASS sources with (H-Ks) $>0.7$ in the RMC, with a field of $2^{\circ} \times 2^{\circ}$ centered at R.A. $=06 \mathrm{~h} 32 \mathrm{~m} 24 \mathrm{~s}$, Dec. $=+04 \mathrm{~d} 25 \mathrm{~m} 00 \mathrm{~s}$ (J2000). Well confined regions of clustered star formation are marked as A, B, C \& D. Contours of the IRAS HIRES $100 \mu \mathrm{m}$ emission are superimposed onto the same plot to further elucidate the spatial consistency of the embedded clusters with the dense clumpy regions. Positions of the 7 minor aggregations identified by Phelps \& Lada (1997) are plotted as pluses. The location of the candidate proto-O stars associated with AFGL 961 is indicated by a solid square. A dashed circle is drawn at the upper right of the plot indicating the location of the young open cluster NGC 2244 and its associated HII region in expansion.

Fig. 2.- Color-color diagrams of the outlined regions of on-going cluster formation. The 2MASS sources that fit our selection criteria are plotted as dots. Diamond symbols indicate the most extreme source in each corresponding region. Components of the conventional binary system AFGL 961 are shown with asterisks, the eastern component of which is further demarcated by an arrow pointing upwards indicating an upper limit detection in the J band and consequently a lower limit to J-H. The third component of the system is denoted as a square. Solid lines represent the loci of the main-sequence dwarfs and giant stars (Bessel \& Brett 1988). The arrow in the upper left of the plot shows a reddening vector of $\mathrm{Av}=5 \mathrm{mag}$ (Rieke \& Lebofsky 1985). The dot-dashed line indicates the locus of dereddened T Tauri stars (Meyer et al. 1997). The dashed lines define the reddening band for normal stars and $\mathrm{T}$ Tauri stars, and are drawn parallel to the reddening vector, with crosses overplotted with an interval corresponding to 5 mag of visual extinction.

Fig. 3.- A color-magnitude diagram of the Region $\mathrm{C}$ cluster and its criteria missed candidate young stellar objects. Sample sources toward Region $\mathrm{C}$ are plotted as dots and the criteria missed sources as pluses. Both components of the massive binary AFGL 961, including the east component that was overlooked by our source selection due to its upper limit detection in the $\mathrm{J}$ band as discussed in $\S 2$, are indicated as asterisks. The third component of the system, AFGL 961 II, is denoted as a square (see also Li \& Smith 2005b). The main-sequence with masses between 0.8 and $120 \mathrm{M} \odot$ is plotted as a solid line (Lejeune \& Schaerer 2001). The slanted line with an arrow at the tip denotes a reddening of $\mathrm{Av}=20$ mag of a A0 type dwarf. The dotted line indicates the reddening track of a B0 type star on the main sequence.

Fig. 4.- a) Distribution of optical depth of the RMC at $100 \mu \mathrm{m}$ derived from IRAS ISSA. The overplotted contours are between $1.13 \times 10^{-4}$ and $1.16 \times 10^{-3}$, with a step of $1.2 \times$ $10^{-3}$. The fragmented interaction shells of the Rosette Nebula with its surroundings is clearly seen as a circular structure, where star formation is more or less emphatically going on. b) Distribution of effective temperature of cold dust in the RMC. The contours are drawn between $20.45 \mathrm{~K}$ and $41.0 \mathrm{~K}$, with a step of $0.41 \mathrm{~K}$. Note the distribution of condensed 
regions with higher cold dust temperature in the south-east of the RMC. This, along with the regulated distribution of embedded sources in this region, shows evidence that the dust temperature can be a key parameter governing the formation of stellar aggregates or clusters.

Fig. 5.- The structured distribution of the sample sources with $(\mathrm{H}-\mathrm{Ks})>0.5$ mag into a tree pattern, schematically illustrated by the thick continuous lines. 


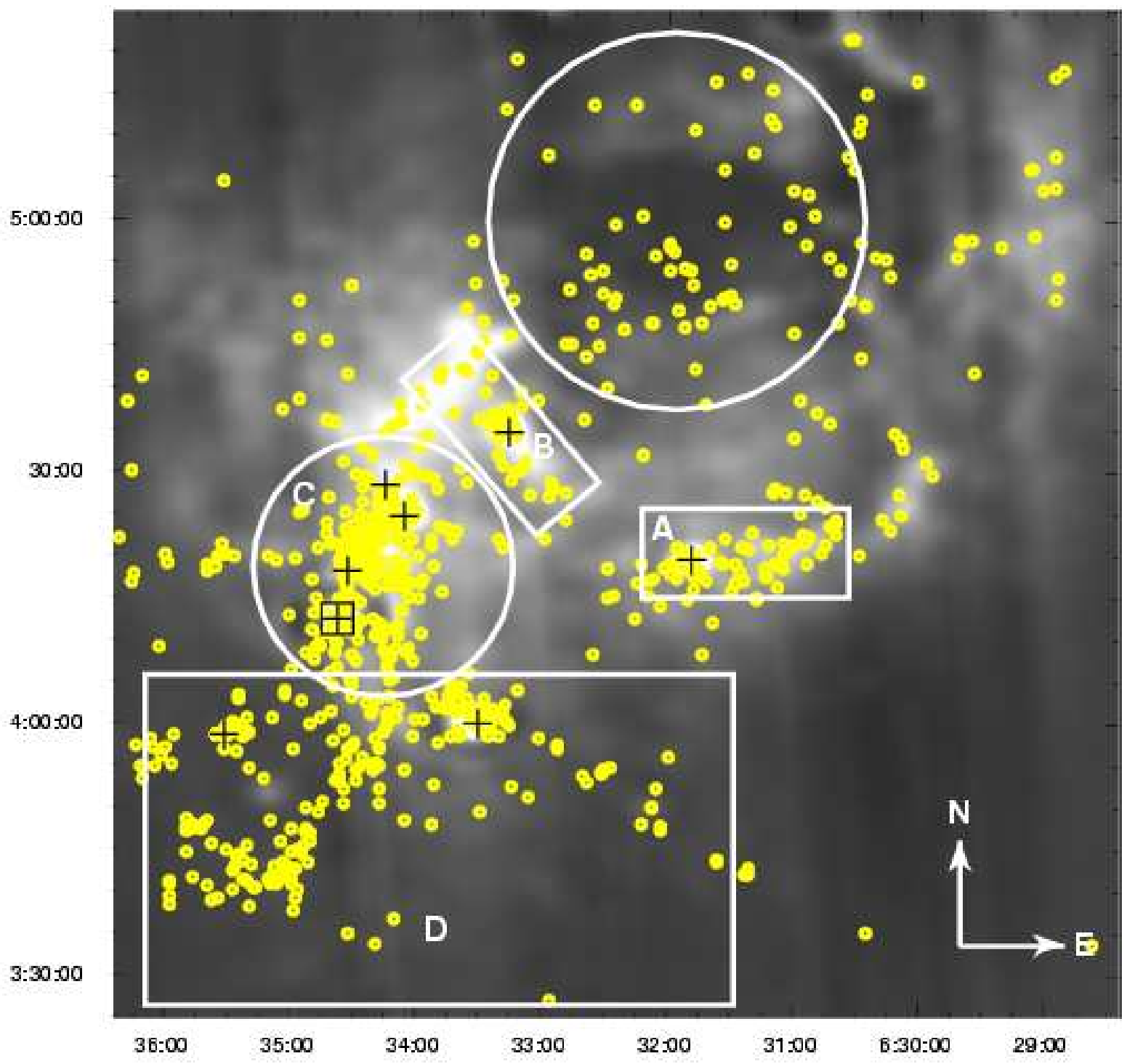



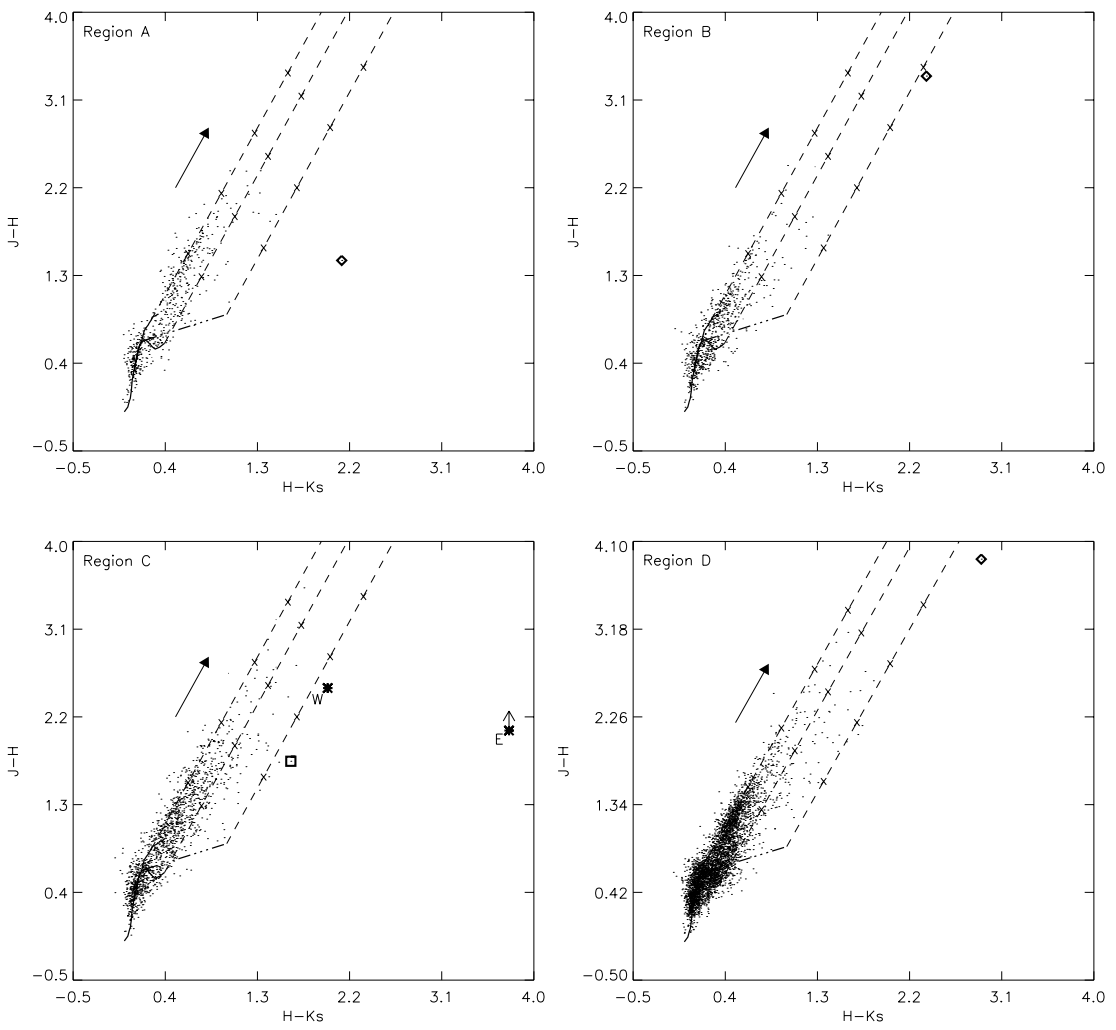


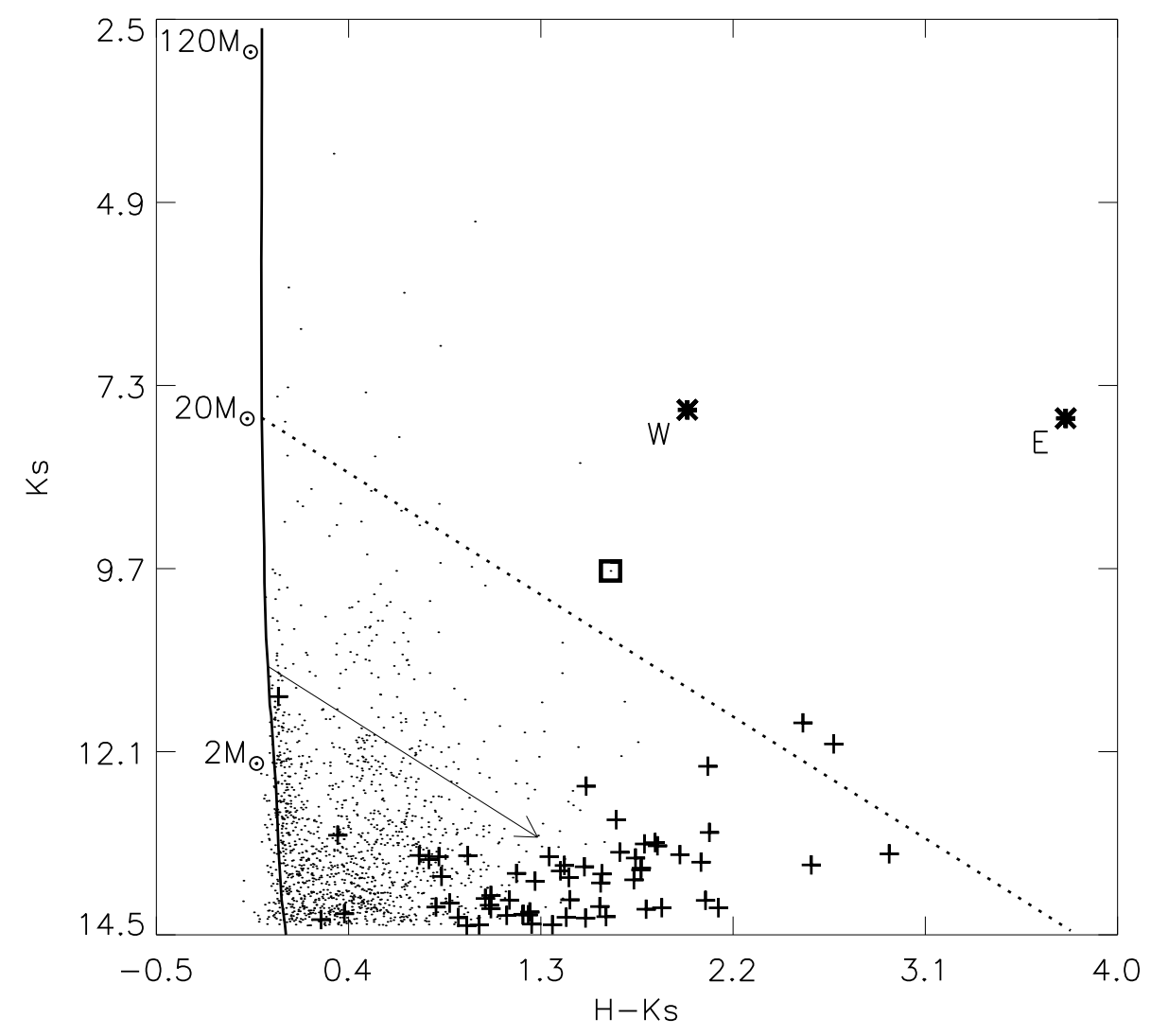



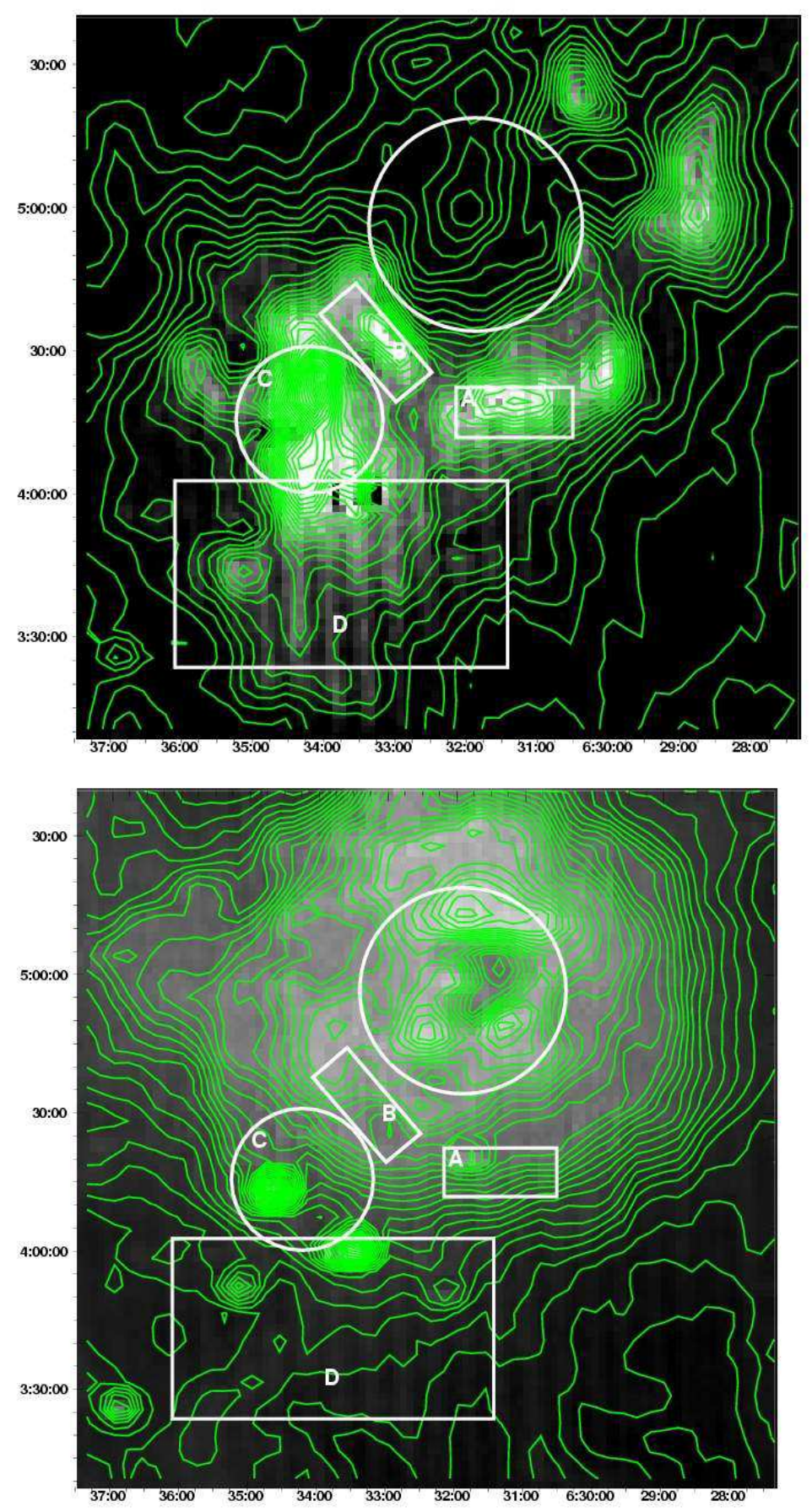


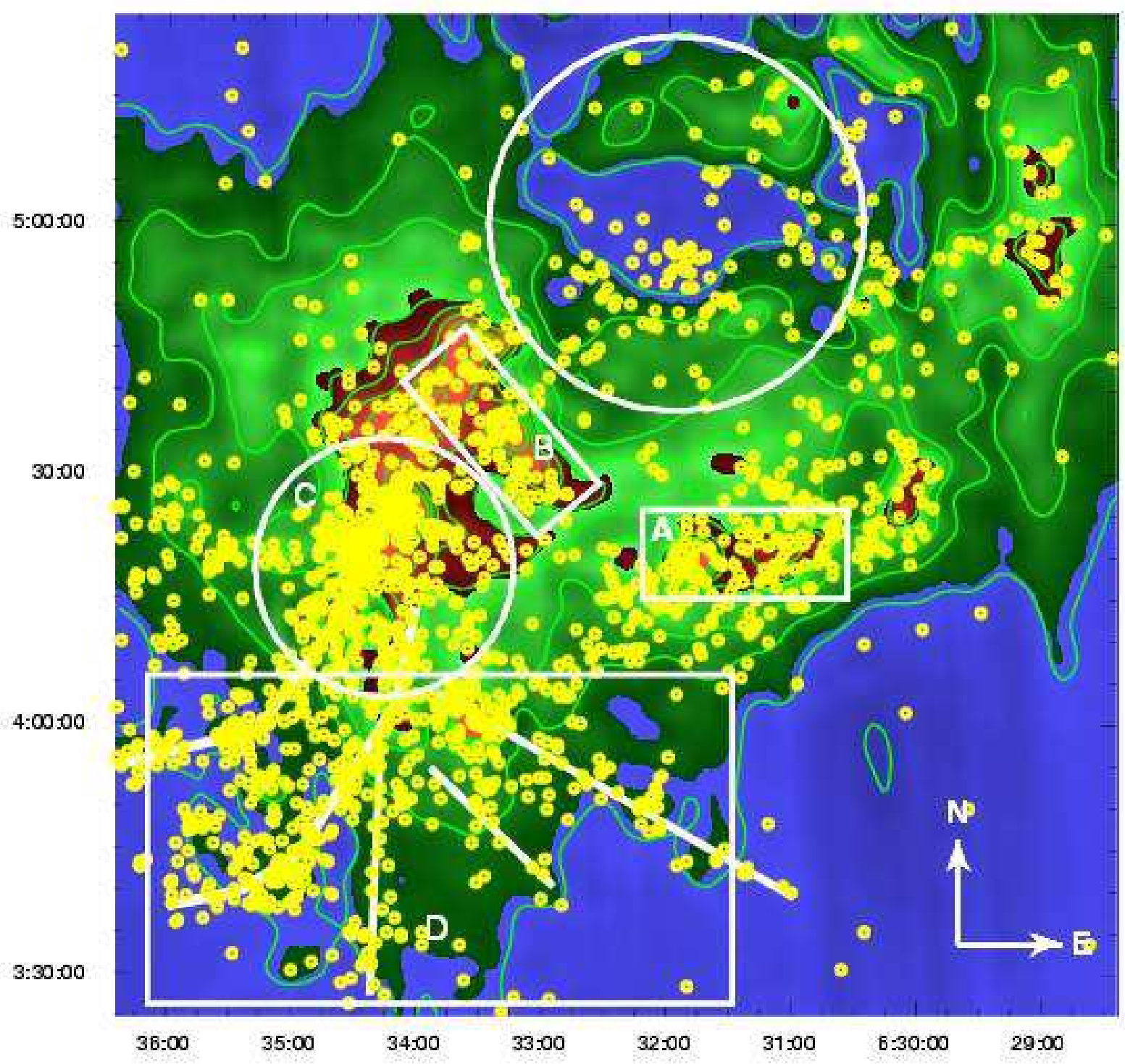


Table 1. Near infrared clusters in the RMC

\begin{tabular}{|c|c|c|c|c|c|c|c|}
\hline Sample & Arbitrary center & Spatial scale & Av & Weighted & Embedded & Evolutionary & Most massive \\
\hline Region & RA (J2000) Dec (J2000) & $(\operatorname{arcmin})$ & (mag) & mean (mag) & population & age (Myr) & object $(\mathrm{M} \odot)$ \\
\hline A & 063128.20041906 .6 & $27.90 \times 15.78$ & $0.2-15.0$ & $7.0 \pm 0.5$ & 41 & $\sim 1$ & 20 \\
\hline B & 063310.80043440 .8 & $7.20 \times 19.40$ & $0.5-19.5$ & $2.5 \pm 0.5$ & 47 & $\sim 1$ & 20 \\
\hline $\mathrm{C}$ & 063418.48041951 .6 & $32.00 \times 32.00$ & $0.5-21.5$ & $4.0 \pm 0.5$ & 123 & $<1$ & 130 \\
\hline $\mathrm{D}$ & 063349.50043004 .5 & $74.25 \times 38.33$ & $0.5-24.5$ & $4.0 \pm 0.5$ & 251 & $<1$ & 50 \\
\hline
\end{tabular}

*The sample region corresponding to each major cluster, an arbitrary central position, spatial extension of each sample area, visual extinction as estimated from the near infrared color-color diagrams, a weighted mean visual extinction toward each field, a lower limit of the embedded stellar population and the mass of the most massive component of each cluster. 